\title{
Interval angioarchitectural evolution of brain arteriovenous malformations following rupture
}

\author{
Hengwei Jin, MD,,2 Stephanie Lenck, MD, ${ }^{1}$ Timo Krings, MD, PhD, ${ }^{1}$ Ronit Agid, MD, ${ }^{1}$ \\ Yibin Fang, MD, ${ }^{1,4}$ Youxiang Li, MD, , ${ }^{2,3}$ Alex Kostynskyy, MD, ${ }^{1}$ Michael Tymianski, MD, PhD, 5,6 \\ Vitor Mendes Pereira, MD, ${ }^{1,5}$ and Ivan Radovanovic, MD, PhD ${ }^{5,6}$
}

\begin{abstract}
1'Division of Neuroradiology, Department of Medical Imaging, Toronto Western Hospital, University Health Network, Toronto, Ontario, Canada; ' 2 epartment of Interventional Neuroradiology, Beijing Neurosurgical Institute and Beijing Tiantan Hospital, Capital Medical University, Beijing, China; ${ }^{3}$ Beijing Engineering Research Center for Interventional Neuroradiology, Beijing, China; ${ }^{4}$ Department of Neurosurgery, Changhai Hospital, Second Military Medical University, Shanghai, China; ${ }^{5}$ Division of Neurosurgery, Department of Surgery, Toronto Western Hospital, University Health Network, University of Toronto, Ontario, Canada; and ${ }^{6}$ Krembil Neuroscience Center, University Health Network, Toronto, Ontario, Canada
\end{abstract}

OBJECTIVE The goal of this study was to describe changes in the angioarchitecture of brain arteriovenous malformations (bAVMs) between acute and delayed cerebral digital subtraction angiography (DSA) obtained after hemorrhage, and to examine bAVM characteristics predicting change.

METHODS This is a retrospective study of a prospective institutional bAVM database. The authors included all patients with ruptured bAVMs who had DSA in both acute and delayed phases, with no interval treatment of their bAVM, between January 2000 and April 2017. The authors evaluated the existence or absence of angioarchitectural changes. Demographic data, radiological characteristics of hemorrhages, and angioarchitectural features of the bAVMs of the two patients' groups were analyzed. Univariate and multivariate logistic analyses were performed to identify predictors of angioarchitectural change.

RESULTS A total of 42 patients were included in the series. Seventeen (40.5\%) patients had angioarchitectural changes including bAVM only visible on the delayed DSA study $(n=8)$, spontaneous thrombosis of the AVM $(n=3)$, or alteration of the size or the opacification of the nidus $(n=6)$. The factors associated with angioarchitectural changes were a small nidus ( $3.8 \pm 7.9 \mathrm{ml}$ vs $6.1 \pm 9.5 \mathrm{ml}, p=0.046)$, a superficial location $(94.1 \%$ vs $5.9 \%, p=0.016)$, and a single superficial draining vein $(58.8 \%$ vs $24.0 \%, p=0.029)$.

CONCLUSIONS Angioarchitectural changes can be seen in $40 \%$ of ruptured bAVMs between the acute- and delayedphase DSA. A small nidus, a superficial location, and a single superficial draining vein were statistically associated with the occurrence of angioarchitectural changes. These changes included either enlargement or spontaneous occlusion of the bAVM, as well as subsequent diagnosis of a bAVM following an initial negative DSA study.

https://thejns.org/doi/abs/10.3171/2018.2.JNS18128

KEYWORDS arteriovenous malformation; angioarchitecture; intracranial hemorrhage; vascular disorders

$\mathrm{T}$ HE risk of recurrent hemorrhage and the consequent high morbidity and mortality rates $(10 \%-30 \%$ and $0 \%-10 \%$, respectively) justify the curative treatment of ruptured brain arteriovenous malformations (bAVMs). ${ }^{8,18}$ However, the optimal timing of such treatment remains unclear. $4,11,21,28,31$ Technical advances in microsurgery and endovascular techniques may have decreased the surgery-related risks of bAVMs and increased the rate of complete occlusion or resection. ${ }^{6,9,23}$ Otherwise, the rate of recurrent hemorrhage is reported to be as high as $6 \%$ in the first year after the onset of bleeding, ${ }^{7}$ encouraging some authors to propose bAVM treatment in the acute phase of hemorrhage. ${ }^{21,28}$ However, the more traditional approach of targeted embolization of

ABBREVIATIONS AHA = American Heart Association; bAVM = brain arteriovenous malformation; DSA = digital subtraction angiography; EVD = external ventricular drain; $\mathrm{ICA}=$ internal carotid artery; ICH = intracerebral hemorrhage; $\mathrm{IVH}=$ intraventricular hemorrhage; $\mathrm{MCA}=$ middle cerebral artery; $\mathrm{mRS}=$ modified Rankin scale; SAH = subarachnoid hemorrhage; $\mathrm{SM}=$ Spetzler-Martin; VA = vertebral artery.

SUBMITTED January 15, 2018. ACCEPTED February 23, 2018.

INCLUDE WHEN CITING Published online July 27, 2018; DOI: 10.3171/2018.2.JNS18128. 
bAVM-associated aneurysms followed by delayed curative treatment (6-12 weeks) is still preferred in most centers ${ }^{31}$ and is recommended by the American Heart Association (AHA). ${ }^{4}$ This last strategy is supported by the lower risk and higher chances of curative treatment at the delayed phase compared to the acute phase. There is also a general perception that the size of the nidus may be underestimated in the acute phase because the bAVM may be compressed by the hematoma. However, the angioarchitectural changes of bAVMs as seen on digital subtraction angiography (DSA) between the acute and delayed phases after hemorrhage have never been systematically studied. The aims of this study were to evaluate and describe these angioarchitectural changes and to determine which bAVMs are most likely to change.

\section{Methods \\ Patient Population}

This study was approved by our institutional ethical review board. Written informed consent was obtained for each patient before all procedures according to institutional protocol. A total of 353 consecutive patients with ruptured bAVMs treated between January 2000 and April 2017 were retrospectively reviewed from our prospective bAVM database. The process of patient inclusion is demonstrated in Fig. 1.

Inclusion criteria were as follows: ruptured cerebral bAVM, including subarachnoid hemorrhage (SAH), intraventricular hemorrhage (IVH), and intracerebral hemorrhage (ICH); and DSA in the acute phase (within the first 7 days after hemorrhage) and in the delayed phase (6 weeks or longer after hemorrhage) ${ }^{4}$ Exclusion criteria were as follows: patients with incomplete demographic information or clinical and/or imaging data; patients with a ruptured flow-related aneurysm of the circle of Willis; patients who underwent neurosurgery (except external ventricular drain [EVD] placement) between the two DSA studies; patients who underwent embolization (except for the target embolization of an associated intranidal aneurysm) between the two DSA studies; and patients who underwent radiosurgery between the two DSA studies.

\section{Clinical Data}

Age, sex, follow-up duration, and the modified Rankin scale (mRS) score at both initial presentation and at last follow-up were retrospectively recorded from the electronic chart of the patients (QuadraMed EPR System, Harris). The need for EVD placement or for a targeted embolization of intranidal aneurysms was recorded. The images of patients who underwent targeted embolization were carefully and independently reviewed by the authors. The patients were included in the study only if all authors thought that the embolization procedure did not significantly modify the angioarchitecture of the bAVM.

\section{Radiological Data}

All patients had at least one CT or MRI study at the time of diagnosis and at least two DSA studies, one in the acute phase and the other during the delayed phase after the hemorrhage (after 6 weeks). Cerebral angiography was

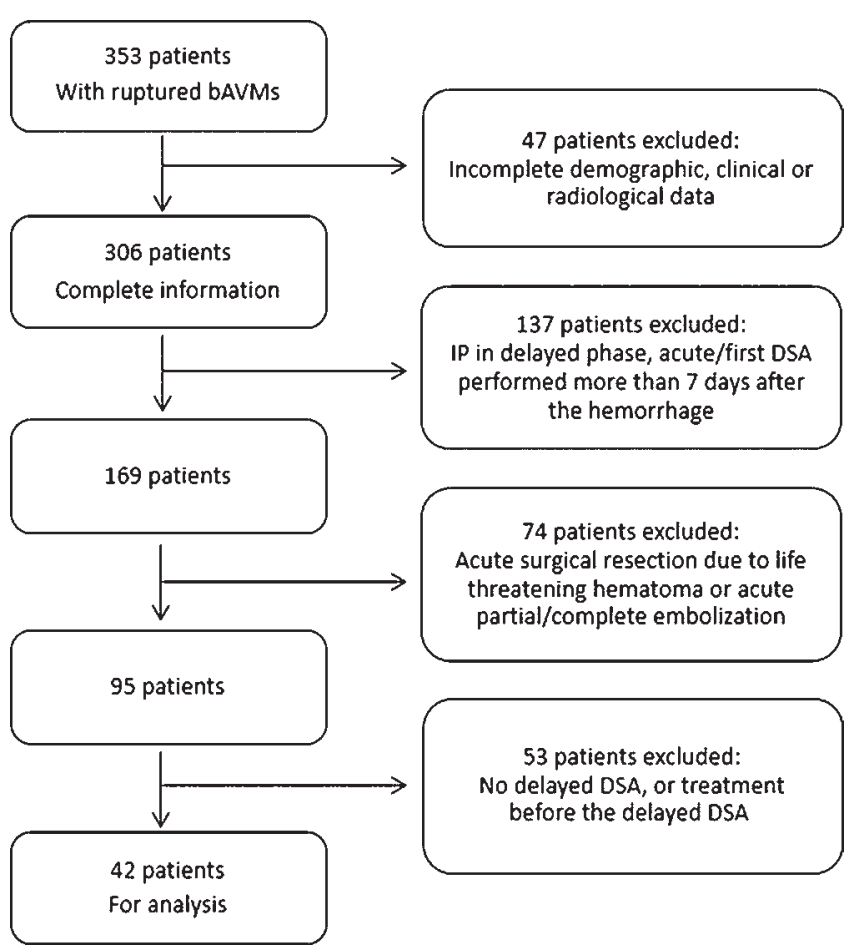

FIG. 1. Flowchart demonstrating the breakdown of 353 patients in the ruptured bAVM database to 42 patients for analysis. IP = initial presentation.

performed in a standardized fashion at both the acute and delayed phases according to institutional protocol. All patients underwent 6-vessel cerebral angiography with biplane angiography, including injection of both internal carotid arteries (ICAs) and external carotid arteries, and of the vertebral arteries (VAs) bilaterally. The delay between the two DSA studies was recorded. Two interventional neuroradiologists (H.J., S.L.) independently reviewed the imaging results of the patients and compared the angioarchitectural features of bAVMs at both the acute and delayed phase on DSA. In the case of significant discordance between the two analyses, the images were reviewed with two other authors (I.R., V.M.P.) and a consensus was found.

\section{Radiological Features of Brain Hemorrhage}

The type of hemorrhage was categorized as $\mathrm{ICH}, \mathrm{SAH}$, and/or IVH. The side and the location of ICH were recorded as supra- or infratentorial, and superficial or deep. Superficial location was defined as the cortex and subcortical white matter. Deep location was defined as the corpus callosum, the thalamus, the basal ganglia, the brainstem, the cerebellar peduncles, and the deep cerebellar nuclei. The volume of the hemorrhage was calculated as (length $\times$ width $\times$ height) $/ 2$ based on the first CT or MRI, and was expressed in milliliters. The initial Fisher grade was recorded.

\section{Angioarchitectural Features of the bAVM}

For bAVM as for ICH, the side, the supra- or infratentorial location, and the superficial or deep locations of the 
TABLE 1. Demographic data and angioarchitectural characteristics in patients with bAVMs

\begin{tabular}{|c|c|c|c|c|}
\hline Items & Total, $n=42$ & Change, $n=17$ & No Change, $n=25$ & $\mathrm{p}$ Value \\
\hline Mean age, yrs & $40.3 \pm 17.1$ & $37.9 \pm 18.0$ & $42.0 \pm 16.6$ & 0.488 \\
\hline Male sex & $24(57.1)$ & $11(64.7)$ & $13(52.0)$ & 0.530 \\
\hline Mean interval btwn acute \& delayed DSA, mos & $9.2 \pm 10.8$ & $7.3 \pm 3.8$ & $10.4 \pm 13.7$ & 0.877 \\
\hline Location of nidus & & & & 0.603 \\
\hline Supratentorial & $35(83.3)$ & $14(82.4)$ & $21(84.0)$ & \\
\hline Infratentorial & $7(16.7)$ & $3(17.6)$ & $4(16.0)$ & \\
\hline Superficial or deep location of nidus & & & & 0.016 \\
\hline Superficial & $31(73.8)$ & $16(94.1)$ & $15(60.0)$ & \\
\hline Deep & $11(26.2)$ & $1(5.9)$ & $10(40.0)$ & \\
\hline Mean vol of nidus, $\mathrm{ml}$ & $5.2 \pm 8.8$ & $3.8 \pm 7.9$ & $6.1 \pm 9.5$ & 0.046 \\
\hline Single feeding artery & $5(11.9)$ & $2(11.8)$ & $3(12.0)$ & 1.000 \\
\hline Single draining vein & $26(61.9)$ & $13(76.5)$ & $13(52.0)$ & 0.195 \\
\hline Single superficial draining vein & $16(38.1)$ & $10(58.8)$ & $6(24.0)$ & 0.029 \\
\hline Deep drainage & $20(47.6)$ & $5(29.4)$ & $15(60.0)$ & 0.066 \\
\hline SM grade & & & & 0.207 \\
\hline$|-| \mid$ & $27(64.3)$ & $13(76.5)$ & $14(56.0)$ & \\
\hline III-V & $15(35.7)$ & $4(23.5)$ & $11(44.0)$ & \\
\hline Type of hemorrhage & & & & 0.177 \\
\hline $\mathrm{ICH} ;-\mathrm{SAH} / \mathrm{IVH}$ & $17(40.5)$ & $6(35.3)$ & $11(44.0)$ & \\
\hline $\mathrm{ICH} ;+\mathrm{SAH} / \mathrm{IVH}$ & $11(26.2)$ & $7(41.2)$ & $4(16.0)$ & \\
\hline SAH \&/or IVH & $14(33.3)$ & $4(23.5)$ & $10(40.0)$ & \\
\hline Mean vol of ICH, ml & $12.67 \pm 18.6$ & $15.4 \pm 20.7$ & $10.8 \pm 17.3$ & 0.210 \\
\hline Initial mRS score & & & & 0.353 \\
\hline$\leq 2$ & $26(61.9)$ & $9(52.9)$ & $17(68.0)$ & \\
\hline$\geq 3$ & $16(38.1)$ & $8(47.1)$ & $8(32.0)$ & \\
\hline EVD \&/or targeted embolization of a pre- or intranidal aneurysm & $9(21.4)$ & $1(5.9)$ & $8(32.0)$ & 0.06 \\
\hline Mean clinical follow-up time, mos & $38.8 \pm 51.2$ & $26.2 \pm 23.6$ & $47.4 \pm 62.6$ & 0.311 \\
\hline mRS score at last follow-up & & & & 0.260 \\
\hline$\leq 2$ & $39(92.9)$ & $17(100.0)$ & $22(88.0)$ & \\
\hline$\geq 3$ & $3(7.1)$ & $0(0.0)$ & $3(12.0)$ & \\
\hline
\end{tabular}

Values are expressed as number of patients (\%) or as the mean \pm SD. Boldface type indicates statistical significance.

nidus were recorded. The volume of the nidus was calculated as (length $\times$ width $\times$ height) $/ 2$ and expressed in milliliters based on the initial MRI or CTA. The SpetzlerMartin (SM) grade was determined for each bAVM..$^{25}$ The venous drainage pattern was considered deep if the venous drainage of the bAVM involved the deep veins. In the posterior fossa, only cerebellar hemispheric veins that drain directly into the straight sinus or the transverse sinus were considered to be superficial. ${ }^{3}$ We differentiated bAVMs with a single feeding artery from those with a single draining vein.

\section{Statistical Analysis}

All statistical analysis was conducted using SPSS 20.0 (IBM Corp.). Patient characteristics were described with frequencies for categorical variables and mean $\pm \mathrm{SD}$ for continuous variables. Categorical variables were compared using the Fisher exact test or the Pearson chi-square test. Continuous variables were compared between groups by using the Student test (normal distribution) or the Mann-
Whitney U-test (nonnormal distribution). Univariate and multivariate logistic analyses were done to assess predictors for angioarchitectural change. A p value $<0.05$ was considered significant.

\section{Results}

\section{Demographic Data}

Demographic data and angioarchitectural characteristics are listed in Table 1. A total of 42 (24 male; 57.1\%) patients were included. Age ranged from 18 to 70 years (mean \pm SD $40.3 \pm 17.1$ years). The mean follow-up of the patients was $38.8 \pm 51.2$ months. Nine (21.4\%) patients received an EVD ( $\mathrm{n}=7,16.7 \%)$ and/or underwent targeted glue embolization for an intranidal aneurysm $(n=3,7.1 \%)$ at the acute phase of bleeding. No patients experienced rebleeding between the two DSA studies. The time between hemorrhage and the first DSA session ranged from 1 to 5 days (mean 1.8 days). The mean time between the two DSA studies (acute and delayed phases) was 9.2 months 

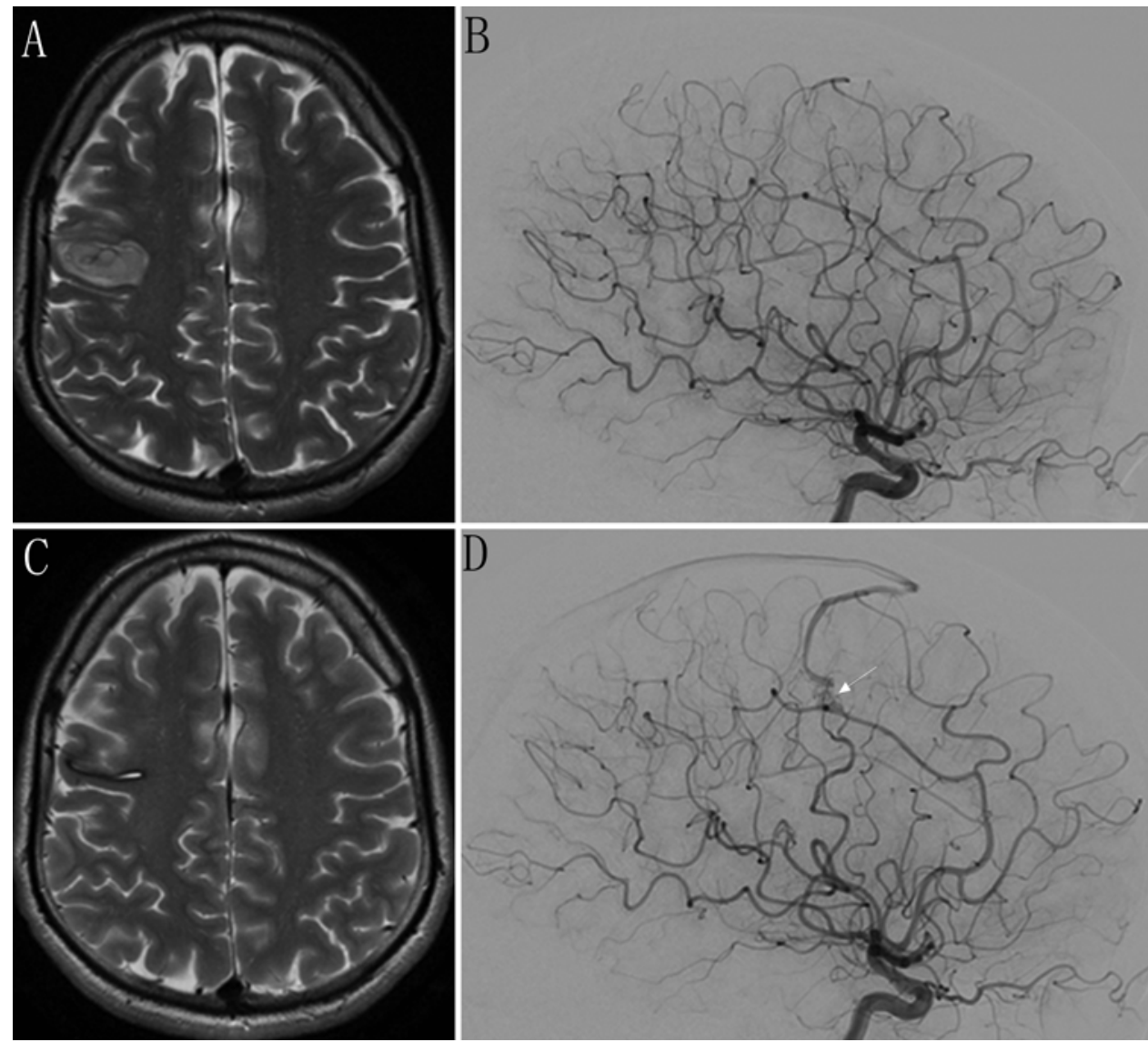

FIG. 2. A: Axial MRI in the acute phase shows hematoma and mass effect in the right frontal lobe. B: Lateral view of right ICA angiography in the acute phase shows no abnormality of the cerebral vasculature. C: Axial MRI in the delayed phase shows hematoma absorption and resolution of mass effect, with no evidence of underlying lesion. D: Lateral view of right ICA angiography in the delayed phase shows a small AVM (arrow) located in the right frontal lobe, supplied by two small precentral branches of the right middle cerebral artery (MCA), with a single superficial draining vein toward the superior sagittal sinus.

(median 6 months). Seventeen $(40.5 \%)$ patients presented with ICH, 14 (33.3\%) with SAH and/or IVH, and 11 (26.2\%) with both ICH and SAH/IVH. The mean volume of the hematoma in patients with ICH was $12.67 \pm 18.6 \mathrm{ml}$. For SAH/IVH, 3 (12\%) were Fisher grade 2, $2(8 \%)$ were grade 3 , and $20(80 \%)$ were grade 4 . The location of the bAVM was superficial in $31(73.8 \%)$ patients, deep in 11 (26.2\%), supratentorial in $35(83.3 \%)$, and infratentorial in 7 (16.7\%). Twenty-six patients (61.9\%) had a single draining vein and $16(38.1 \%)$ had a single superficial draining vein. Twenty-seven (64.3\%) bAVMs were classified as SM grade I-II, and 15 (35.7\%) as SM grade III-V.

\section{Angioarchitectural Changes}

The clinical and demographic features of the patients as well as the angioarchitectural characteristics of their bAVMs were compared regarding the presence or absence of angioarchitectural changes over time. This comparison is summarized in the Table 1 . Seventeen (40.5\%) patients showed angioarchitectural changes between the acute and delayed DSA. In 8 patients, the initial DSA study was negative, and the BAVM was diagnosed with the delayed DSA (Fig. 2). All of those patients $(n=8)$ had ICH $( \pm$ $\mathrm{SAH} / \mathrm{IVH})$ at presentation. In 6 patients, the bAVM was bigger or more opacified at the delayed phase compared to the acute phase (Fig. 3). In 3 patients, the DSA study was positive in the acute phase but negative in the delayed phase. The spontaneous cure of the bAVM was confirmed 6 months to 1 year after the second DSA study with cerebral MRI (Fig. 4).

The bAVMs that exhibited angioarchitectural changes were smaller $(3.8 \pm 7.9$ vs $6.1 \pm 9.5 \mathrm{ml}, \mathrm{p}=0.046)$, with a single superficial draining vein $(58.8 \%$ vs $24 \%, \mathrm{p}=0.029)$, and more likely to be superficial $(94.1 \%$ vs $60.0 \%, \mathrm{p}=$ 0.016) (Table 1). Univariate logistic analysis demonstrated that superficial location $(\mathrm{p}=0.033)$ and a single superficial draining vein $(\mathrm{p}=0.026)$ were statistically significant in predicting angioarchitectural changes (Table 2).

\section{Discussion}

In our study, 40\% (17/42) of ruptured bAVMs demonstrated angioarchitectural changes between the acute and the delayed DSA after hemorrhage, including either enlargement or spontaneous occlusion of the bAVM as well as subsequent diagnosis of a bAVM following an initial negative DSA study. A single superficial vein, a small nidus, and a superficial location were statistically associated 

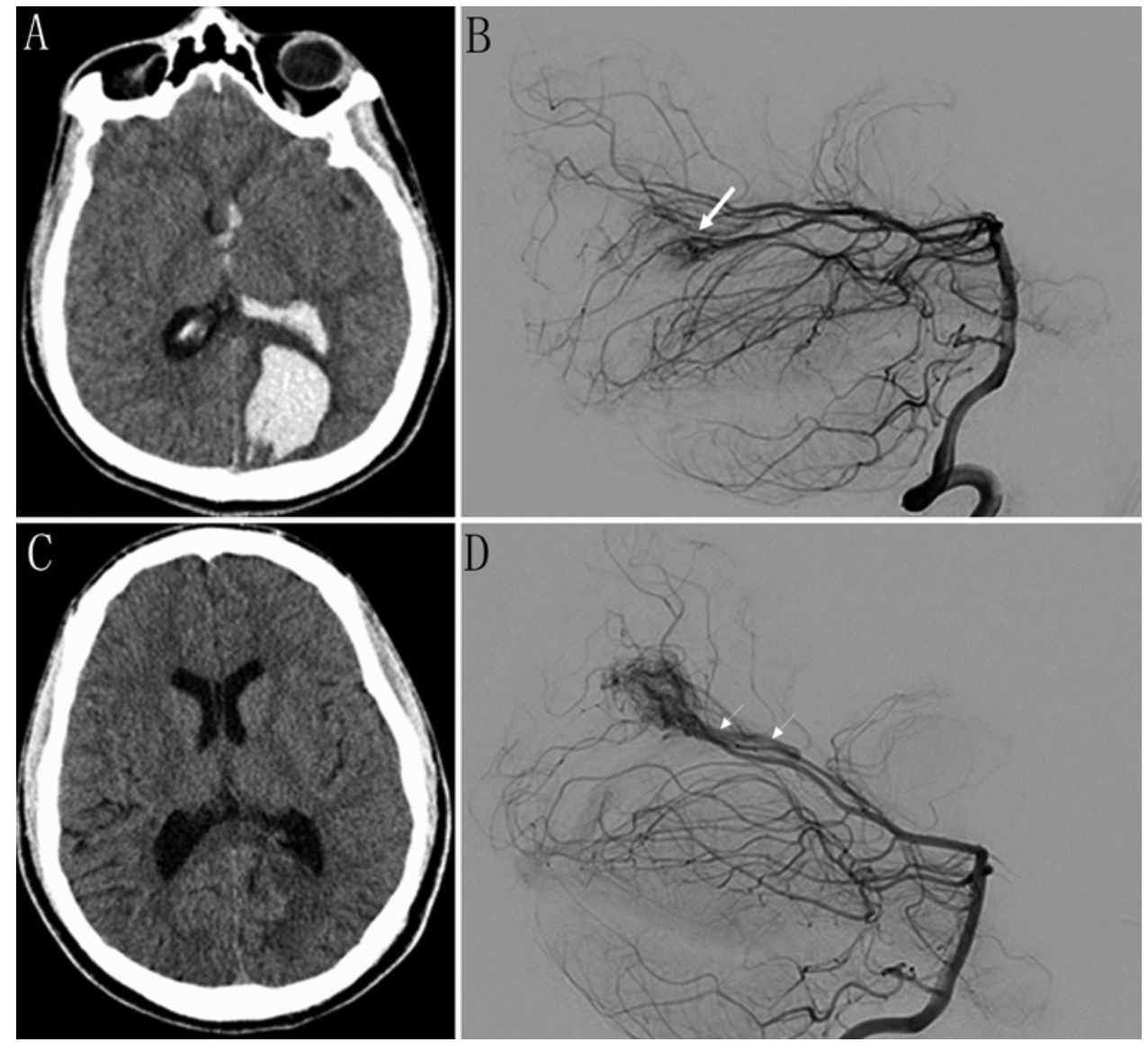

FIG. 3. A: Axial CT in the acute phase shows hematoma and mass effect in the left occipital lobe, as well as IVH. B: Lateral view of left VA angiography in the acute phase shows a small AVM (arrow) located in the left occipital lobe, supplied by branches of the left posterior cerebral artery. The drainage vein is not well demonstrated at this late arterial phase. C: Axial CT in the delayed phase shows hematoma absorption and resolution of mass effect. D: Lateral view of left VA angiography in the delayed phase shows that the AVM is much larger and more readily opacified compared with the acute-phase DSA study. Deep drainage vein is now more obvious at this late arterial phase (arrows).

with those angioarchitectural changes. New appearance of a bAVM on the delayed DSA study and increase in size of the bAVM between the acute and the delayed DSA occurred in 19\% (8/42) and 14\% (6/42) patients, respectively. The pathophysiology is unclear, but the most likely mechanism seems to be the compression of the nidus and/or the draining vein by the hematoma, or the increased intracranial pressure at the acute phase of hemorrhage. Small superficial vascular malformations with a single superficial draining vein appear to be more vulnerable because they are pressed against the rigid surface of the calvaria. The resorption of the hematoma after several weeks may lead to the decompression of the nidus, and disclosure of parts of or the entire bAVM. ${ }^{29,30}$ This observation gives support to the indication for delayed angiography in patients with unexplained intracranial bleeding (especially ICH) and suspicion of underlying vascular lesion to completely eliminate the diagnosis of a small ruptured bAVM.

Spontaneous occlusion of a bAVM has previously been reported to occur in less than $2 \%$ of patients. ${ }^{12,27}$ We found a $7 \%(3 / 42)$ rate of such phenomena in our subgroup of patients. Hemorrhagic presentation, small nidus, and a single draining vein have previously been reported as the main characteristics associated with such a phenomenon, and our results support these findings., ${ }^{1,20,22}$ Sawlani et al. reported a case of a spontaneous occlusion of an unruptured bAVM with a radiologically documented thrombosis of the single draining vein of the bAVM. ${ }^{22}$ Therefore, two mechanisms can be involved in the spontaneous cure of bAVMs after hemorrhage. On one hand, a spontaneous thrombosis of the draining vein may lead to both bleeding and subsequent cure of the malformation. On the other hand, the mass effect of the hematoma may lead to compression of the nidus and/or the draining vein and thus to the cure of the bAVM. ${ }^{1,16,19}$ In the literature, $3 \%$ of spontaneous occluded bAVMs recurred after several months or many years, suggesting that long-term follow-up vascular imaging may still be required in those patients. ${ }^{13,17}$

Except for ruptured bAVMs requiring surgical evacuation of the hematoma, it is still controversial whether to perform curative treatment of the bAVM at the acute or the delayed phase (i.e., 6-12 weeks posthemorrhage). Recently, some authors reported curative treatment either by resection or by Onyx embolization in the acute phase of the hemorrhage to avoid early rebleeding. ${ }^{11,28}$ However, the traditional approach of targeted embolization of an 

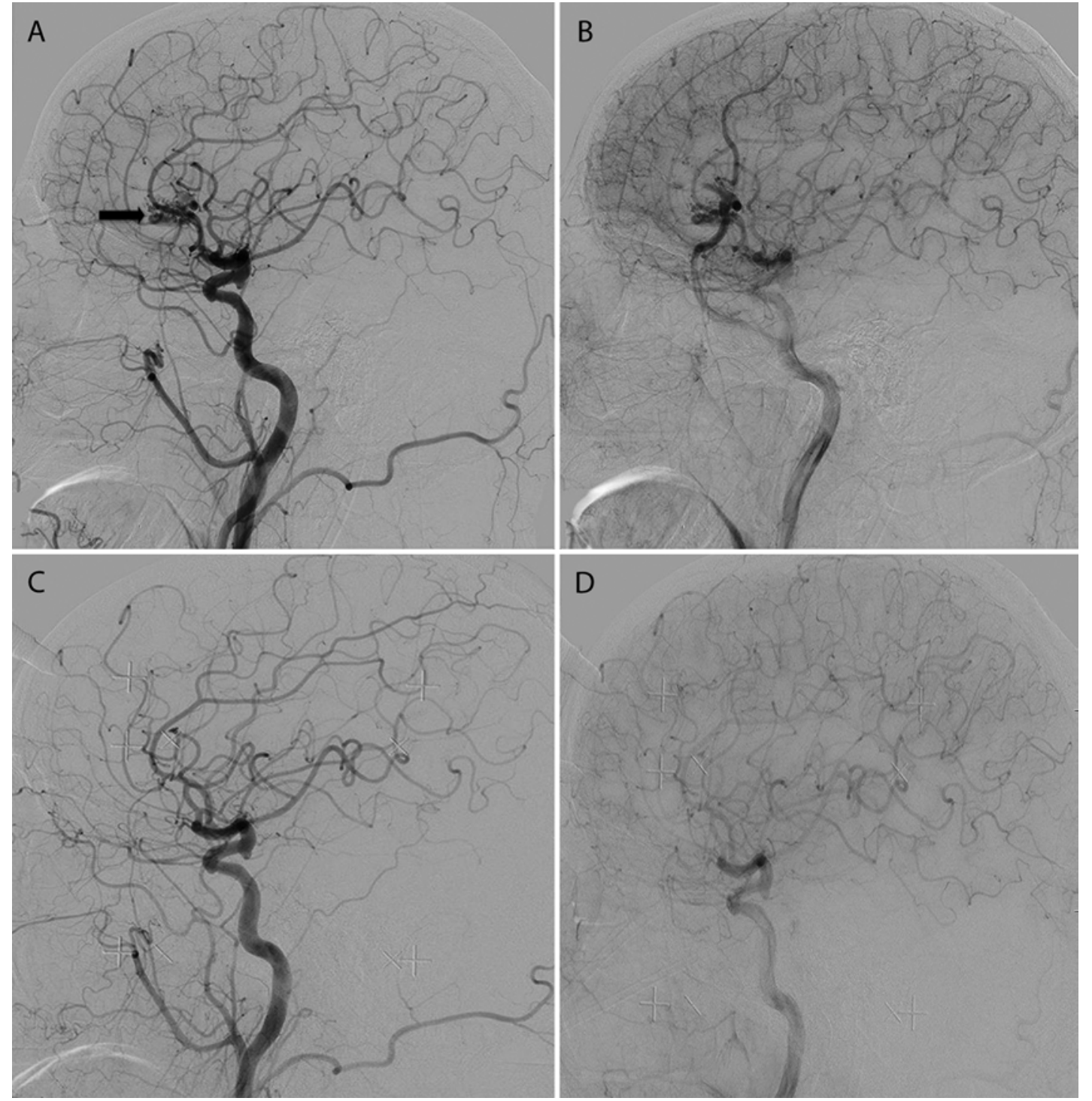

FIG. 4. A and B: Lateral views of left ICA angiography in the acute phase shows a small AVM (arrow) located in the left temporal lobe, supplied by temporal branches of left MCA, with superficial venous drainage. C and D: Lateral views of left ICA angiography in the delayed phase shows no remaining evidence of bAVM. The small temporal AVM has spontaneously thrombosed.

intra- or prenidal aneurysm followed by delayed curative treatment of the bAVM is still practiced in most centers ${ }^{2,10}$ and is recommended by the AHA. ${ }^{4}$ It is well known that delayed surgery may facilitate bAVM resection because it allows the patient to recover from the hemorrhage, the edema to resolve, and the clot to liquefy. ${ }^{4}$ Otherwise, the prothrombotic state induced by platelet activation, as well as the limitation in the administration of anticoagulant drugs at the acute phase of intracranial hemorrhage, may significantly increase the risk of thrombotic complications during embolization. ${ }^{5}$ According to our results, some malformations may actually be larger than apparent on the initial DSA study because of their compression. In those cases, there is a theoretical risk of postoperative bleeding, because the underestimation of the real size of the nidus may lead to partial obliteration (or resection) of the nidus with complete obliteration (or sacrifice) of the draining vein(s). Finally, we showed that a significant portion (7.1\%) of ruptured bAVMs may disappear spontaneously, suggesting that a curative treatment of those lesions at the acute phase of hemorrhage may be futile.

Therefore, both surgery and embolization seem to be safer in the delayed phase of bleeding. However, the risk of early rebleeding has to be considered given its high morbidity and mortality outcome, and the benefit-risk balance should be precisely evaluated when facing an acutely ruptured bAVM. Several observational studies have shown a rate of recurrent hemorrhage as high as $6 \%$ in the first year after the onset of bleeding, ${ }^{7}$ encouraging some authors to propose curative embolization of ruptured bAVMs in the acute phase of the bleeding. ${ }^{21,28}$ The angioarchitecture of the bAVM was not considered in those series, however, even though the presence of a weak point in the malformation is known to significantly increase the hemorrhagic 
TABLE 2. Univariate and multivariate logistic analysis for indicators of angioarchitectural change

\begin{tabular}{|c|c|c|c|c|c|c|}
\hline \multirow[b]{2}{*}{ Characteristic } & \multicolumn{3}{|c|}{ Univariate Logistic Analysis } & \multicolumn{3}{|c|}{ Multivariate Logistic Analysis } \\
\hline & OR & $95 \% \mathrm{Cl}$ & $p$ Value & OR & $95 \% \mathrm{Cl}$ & $\mathrm{p}$ Value \\
\hline Vol $<1 \mathrm{ml}$ & 3.250 & $0.820-12.880$ & 0.093 & 1.022 & $0.905-1.154$ & 0.731 \\
\hline Superficial location & 10.667 & $1.214-93.699$ & 0.033 & 9.401 & $0.688-128.40$ & 0.093 \\
\hline SM grade I-II & 2.554 & $0.648-10.059$ & 0.180 & 0.821 & $0.093-7.261$ & 0.859 \\
\hline Single superficial draining vein & 0.4524 & $1.194-17.146$ & 0.026 & 0.718 & $0.147-11.068$ & 0.691 \\
\hline Single draining vein & 3.000 & $0.764-11.783$ & 0.115 & 3.881 & $0.527-28.590$ & 0.183 \\
\hline Vol of $\mathrm{ICH}$ & 1.013 & $0.980-1.048$ & 0.444 & 1.011 & $0.972-1.052$ & 0.587 \\
\hline
\end{tabular}

Boldface type indicates statistical significance.

risk because it is usually the cause of the bleeding. ${ }^{14,24,26}$ Therefore, targeted embolization of an intra- or prenidal aneurysm of a bAVM in the acute phase may significantly reduce the hemorrhagic risk and is usually safer than a curative treatment of the lesion..$^{10,14,15}$ Although this strategy may limit the possibilities for future embolization by occluding a large feeding artery of the bAVM, it does not limit the possibilities for subsequent resection or radiosurgery.

\section{Limitations of the Current Study}

There are some limitations to this study. First, this is a retrospective study. However, our database is prospectively collected to assure completeness of the data and to prevent inclusion bias.

Second, only a small portion of the overall number of patients with ruptured bAVMs was analyzed in our study (42/353). However, the purpose of the investigation reported here was to evaluate the spontaneous evolution of the angioarchitecture of bAVMs following rupture and not to evaluate our therapeutic strategy. We thus had very selective radiological inclusion criteria in order to minimize the biases linked with the treatment or with insufficient radiological data. The resulting sample size of this study remains nevertheless a limitation.

Finally, the interval between the initial and delayed DSA in our study is longer than that recommended by the AHA, which defines the delayed phase as 6-12 weeks after the initial bleed. This can be explained by the fact that our study was retrospective - and although a 3-month delay for the delayed DSA following hemorrhage is our general rule, the therapeutic management may be more difficult to plan in real-world clinical practice.

\section{Conclusions}

Angioarchitectural changes can be seen in $40 \%$ of ruptured bAVMs between the acute- and delayed-phase DSA. A small nidus, a superficial location, and a single superficial draining vein were statistically associated with those angioarchitectural changes. These changes included either enlargement or spontaneous occlusion of the bAVM, as well as subsequent diagnosis of a bAVM following an initial negative DSA study. These findings support the traditional strategy of acute targeted embolization of an intra- or prenidal aneurysm followed by delayed curative treatment of the bAVM itself, especially for small and superficial bAVMs.
The timing of the delayed DSA and treatment reflects the time required for the resorption of the hematoma (i.e., 6-12 weeks). Otherwise, our study supports the indication for a delayed DSA session in patients with intracranial bleeding (especially ICH) and a suspicion of an underlying vascular lesion, even if the initial DSA study is negative.

\section{Acknowledgments}

Dr. Jin received a grant from the Beijing Municipal Health Bureau: Special Research Project of Capital Health Development, China (grant no. 2016-1-1075). Dr. Lenck received a grant from Assistance Publique des Hôpitaux de Paris, France: Bourse de mobilité APHP.

\section{References}

1. Abdulrauf SI, Malik GM, Awad IA: Spontaneous angiographic obliteration of cerebral arteriovenous malformations. Neurosurgery 44:280-288, 1999

2. Alexander MD, Cooke DL, Hallam DK, Kim H, Hetts SW, Ghodke BV: Less can be more: targeted embolization of aneurysms associated with arteriovenous malformations unsuitable for surgical resection. Interv Neuroradiol 22:445-451, 2016

3. Atkinson RP, Awad IA, Batjer HH, Dowd CF, Furlan A, Giannotta SL, et al: Reporting terminology for brain arteriovenous malformation clinical and radiographic features for use in clinical trials. Stroke 32:1430-1442, 2001

4. Derdeyn CP, Zipfel GJ, Albuquerque FC, Cooke DL, Feldmann E, Sheehan JP, et al: Management of brain arteriovenous malformations: a scientific statement for healthcare professionals from the American Heart Association/American Stroke Association. Stroke 48:e200-e224, 2017

5. Frontera JA, Provencio JJ, Sehba FA, McIntyre TM, Nowacki AS, Gordon E, et al: The role of platelet activation and inflammation in early brain injury following subarachnoid hemorrhage. Neurocrit Care 26:48-57, 2017

6. Gong SF, Wang XB, Liao YQ, Jiang TP, He JB, Wang XJ, et al: Microsurgery for ruptured cerebellar arteriovenous malformations. Eur Rev Med Pharmacol Sci 19:2597-2602, 2015

7. Halim AX, Johnston SC, Singh V, McCulloch CE, Bennett JP, Achrol AS, et al: Longitudinal risk of intracranial hemorrhage in patients with arteriovenous malformation of the brain within a defined population. Stroke 35:1697-1702, 2004

8. Kim H, Al-Shahi Salman R, McCulloch CE, Stapf C, Young WL: Untreated brain arteriovenous malformation: patientlevel meta-analysis of hemorrhage predictors. Neurology 83:590-597, 2014 
9. Kondo R, Matsumoto Y, Endo H, Miyachi S, Ezura M, Sakai $\mathrm{N}$ : Endovascular embolization of cerebral arteriovenous malformations: results of the Japanese Registry of Neuroendovascular Therapy (JR-NET) 1 and 2. Neurol Med Chir (Tokyo) 54 (Suppl 2):54-62, 2014

10. Krings T, Hans FJ, Geibprasert S, Terbrugge K: Partial "targeted" embolisation of brain arteriovenous malformations. Eur Radiol 20:2723-2731, 2010

11. Kuhmonen J, Piippo A, Väärt K, Karatas A, Ishii K, Winkler $\mathrm{P}$, et al: Early surgery for ruptured cerebral arteriovenous malformations. Acta Neurochir Suppl 94:111-114, 2005

12. Leung KM, Agid R, terBrugge K: Spontaneous regression of a cerebral arteriovenous malformation in a child with hereditary hemorrhagic telangiectasia. Case report. J Neurosurg 105 (5 Suppl):428-431, 2006

13. Matano F, Murai Y, Nakagawa S, Wada T, Kitamura T, Teramoto A: Spontaneous angiographic regression of cerebral arteriovenous malformations: angiographic disappearance is not the real cure. Turk Neurosurg 25:168-173, 2015

14. Meisel HJ, Mansmann U, Alvarez H, Rodesch G, Brock M, Lasjaunias P: Cerebral arteriovenous malformations and associated aneurysms: analysis of 305 cases from a series of 662 patients. Neurosurgery 46:793-802, 2000

15. Meisel HJ, Mansmann U, Alvarez H, Rodesch G, Brock M, Lasjaunias P: Effect of partial targeted N-butyl-cyano-acrylate embolization in brain AVM. Acta Neurochir (Wien) 144:879-888, 2002

16. Minakawa T, Tanaka R, Koike T, Takeuchi S, Sasaki O: Angiographic follow-up study of cerebral arteriovenous malformations with reference to their enlargement and regression. Neurosurgery 24:68-74, 1989

17. Mizutani T, Tanaka H, Aruga T: Total recanalization of a spontaneously thrombosed arteriovenous malformation. Case report. J Neurosurg 82:506-508, 1995

18. Ondra SL, Troupp H, George ED, Schwab K: The natural history of symptomatic arteriovenous malformations of the brain: a 24-year follow-up assessment. J Neurosurg 73:387391, 1990

19. Panciani PP, Fontanella M, Carlino C, Bergui M, Ducati A: Progressive spontaneous occlusion of a cerebellar AVM: pathogenetic hypothesis and review of literature. Clin Neurol Neurosurg 110:502-510, 2008

20. Patel MC, Hodgson TJ, Kemeny AA, Forster DM: Spontaneous obliteration of pial arteriovenous malformations: a review of 27 cases. AJNR Am J Neuroradiol 22:531-536, 2001

21. Pavesi G, Rustemi O, Berlucchi S, Frigo AC, Gerunda V, Scienza R: Acute surgical removal of low-grade (SpetzlerMartin I-II) bleeding arteriovenous malformations. Surg Neurol 72:662-667, 2009

22. Sawlani V, Handique A, Phadke RV: Spontaneous regression of cerebral AVM due to thrombosis of draining vein-angiographic and MRI demonstration. J Neurol Sci 223:195-198, 2004

23. Schramm J, Schaller K, Esche J, Boström A: Microsurgery for cerebral arteriovenous malformations: subgroup out- comes in a consecutive series of 288 cases. J Neurosurg 126:1056-1063, 2017

24. Shotar E, Pistocchi S, Haffaf I, Bartolini B, Jacquens A, Nouet A, et al: Early rebleeding after brain arteriovenous malformation rupture, clinical impact and predictive factors: a monocentric retrospective cohort study. Cerebrovasc Dis 44:304-312, 2017

25. Spetzler RF, Martin NA: A proposed grading system for arteriovenous malformations. J Neurosurg 65:476-483, 1986

26. Stefani MA, Porter PJ, terBrugge KG, Montanera W, Willinsky RA, Wallace MC: Angioarchitectural factors present in brain arteriovenous malformations associated with hemorrhagic presentation. Stroke 33:920-924, 2002

27. Stetler WR, Polikfa AJ, Fox WC, Hoh B: Rapid, spontaneous obliteration of intracranial arteriovenous malformation. J Clin Neurosci 34:210-213, 2016

28. van Rooij WJ, Jacobs S, Sluzewski M, Beute GN, van der Pol B: Endovascular treatment of ruptured brain AVMs in the acute phase of hemorrhage. AJNR Am J Neuroradiol 33:1162-1166, 2012

29. Wakai S, Kumakura N, Nagai M: Lobar intracerebral hemorrhage. A clinical, radiographic, and pathological study of 29 consecutive operated cases with negative angiography. J Neurosurg 76:231-238, 1992

30. Willinsky RA, Fitzgerald M, TerBrugge K, Montanera W, Wallace M: Delayed angiography in the investigation of intracerebral hematomas caused by small arteriovenous malformations. Neuroradiology 35:307-311, 1993

31. Zacharia BE, Vaughan KA, Jacoby A, Hickman ZL, Bodmer D, Connolly ES Jr: Management of ruptured brain arteriovenous malformations. Curr Atheroscler Rep 14:335-342, 2012

\section{Disclosures}

The authors report no conflict of interest concerning the materials or methods used in this study or the findings specified in this paper.

\section{Author Contributions}

Conception and design: Radovanovic, Jin, Lenck, Krings, Pereira. Acquisition of data: Jin. Analysis and interpretation of data: Radovanovic, Jin, Lenck. Drafting the article: Radovanovic, Lenck, Pereira. Critically revising the article: Radovanovic, Jin, Lenck, Krings, Agid, Fang, Li, Tymianski, Pereira. Reviewed submitted version of manuscript: all authors. Approved the final version of the manuscript on behalf of all authors: Radovanovic. Statistical analysis: Jin, Lenck. Administrative/technical/material support: Kostynskyy. Study supervision: Radovanovic, Lenck, Pereira.

\section{Correspondence}

Ivan Radovanovic: Toronto Western Hospital, University Health Network and University of Toronto, Toronto, ON, Canada. ivan. radovanovic@uhn.ca. 